\title{
Target maneuver onset time estimation via significance test of acoustic feature difference
}

\author{
Tang Jiansheng ${ }^{1, a}$, Zhang Yongxin ${ }^{2}$, Jiang Xiangdong ${ }^{1}$ \\ ${ }^{1}$ Science and Technology on Underwater Acoustic Antagonizing Laboratory, Beijing 100036, China \\ ${ }^{2}$ No.92330 unit of PLA, Qindao 266102, China \\ aTangjs.npu @gmail.com
}

Keywords: target maneuver; onset time estimation; acoustic feature

\begin{abstract}
An acoustic feature change detecting based method is proposed in the paper to estimate the target maneuver onset time. Form the statistical analysis of many acoustic features of different targets, the distribution of a certain estimated feature value in a single motion state is approximately normal. Base on the normal distribution, we defined the acoustic feature difference index to select the most sensitive feature to the change of target motion state. Using the feature, we proposed an online method to estimate the target maneuver onset time by testing the significance of acoustic feature difference. Experiment results show that the proposed method is more rapid to estimate the maneuver onset time after the target maneuver occurs.
\end{abstract}

\section{Introduction}

Rapid and robust target maneuver onset time detection is crucial to obtain comparable target tracking accuracy [1-6]. Many algorithms and techniques have been developed to detect maneuvers. Most of the detectors attempt to identify a maneuver by predicting the expected target position and comparing it to the measured track. The difference between the predicted position and the measured named innovation. Ref. [6] presents six traditional and two novel maneuver detection algorithms. They are all innovation-based. These methods are quite slow. In the radar area, it typically requires a few seconds to detect a maneuver. But in the sonar area the observer is normally far from the target and the bearing of target is changed very slowly, so it typically requires several minutes to detect a maneuver. It is not acceptable for many practical situations in sonar system.

Feature-based maneuver detection techniques have been proposed in radar area using radar glint noise [7] and range-Doppler images [8]. In fact, radar target feature retains strong link with target motion mode. Compared with innovation-based techniques, the feature-based technique is fast response and more reliable.

In this paper, we exploited the problem using the acoustic features of ship's radiated noise. Since the features of ship's radiated noise have direct link with target motion state, the detector is devised by detecting the acoustic feature differences. Form the ship's radiated noise, we can extract many features. Most of the features are changed during maneuvers, but some of the features are more sensitive to target motion state. We defined the acoustic feature difference index (AFDI) to select the most sensitive feature, and use it to develop the detector by testing the significance of acoustic feature difference. Some experiments were carried out to illustrate the capability of the proposed detector. Experiment results show that the proposed method is more rapid to estimate the maneuver onset time after the target maneuver occurs.

\section{Distribution of the estimated feature value}

In the practical situation, we get the target's radiated noise $s(t)$ in real time. The fix length window can be used to get signal segments with certain duration $T$, such as $0.5 \mathrm{~s}$. Using each signal segment, many features can be extracted by different methods. We use $f_{k}^{l}$ representing the lth feature value of these extracted features estimated by the $k$ th signal segment. Assuming the target's motion state is not change in the $k=1, \cdots, K$, the lth feature value set can be given by 


$$
f^{l}=\left\{f_{k}^{l}, k=1, \cdots K\right\}
$$

According to the central limit theorem, the distribution of $f_{k}^{l}$ is approximately normal. Its mean and variance can be estimated by the feature value set $f^{l}$ :

$$
\begin{aligned}
& \overline{f^{l}}=\frac{1}{K} \sum_{k=1}^{K} f_{k}^{l} \\
& \sigma_{f^{l}}^{2}=\frac{1}{K} \sum_{k=1}^{K}\left[f_{k}^{l}-\overline{f^{l}}\right]
\end{aligned}
$$

We also can see the difference of feature value caused by the same speed change is not consistent. The speed change can make the SWS change more than the NLS, so the SWS can distinguish the speed change better than NLS. We introduce the acoustic feature difference index (AFDI) to select the most sensitive feature, which defined by

$$
\gamma\left(f^{l}, v_{1}, v_{2}\right)=\frac{\left[\overline{f^{l}}\left(v_{1}\right)-\overline{f^{l}}\left(v_{2}\right)\right]^{2}}{\sigma_{f^{l}}^{2}\left(v_{1}\right)+\sigma_{f^{l}}^{2}\left(v_{2}\right)}
$$

If the difference of the mean of certain feature values at two speeds is larger, and the variance of each feature values is smaller in the same time, the AFDI will be larger for the feature. That's mean the feature can distinguish the speed change better than other features. We can use the feature which has largest AFDI to develop the detector to improve the computational efficiency.

\section{Target maneuver onset time detection}

Denote the present time by $k$ and assume that the target starts maneuvering at time $K$. That is, in the $k=1, \cdots, K$, the target motion state is not change. The feature value $f_{k}^{l} \quad(k=1, \cdots, K)$ is approximately normal $f_{k}^{l} \sim N\left(\overline{f^{l}}, \sigma_{f^{l}}^{2}\right)$. After the time $K$, the target motion state may be changed. We defined the test feature value set use the $S$ signal segments after $K$

$$
\tilde{f}^{l}=\left\{f_{k}^{l}, k=K+m, \cdots, K+m+S\right\}
$$

where $\mathrm{m}$ is a given constant, representing the constant delay after $K$. The reason using $\mathrm{m}$ is that some features are not change immediately after the change of target motion state occurs for underwater ships, such as acoustic feature caused by cavitations noise. From the test feature set, we get its mean denoted by $\overline{f_{T}^{l}}$.

If the target is not maneuver in the period $k=K+m, \cdots, K+m+S, \overline{f_{T}^{l}}$ will be close to the $\overline{f^{l}}$, otherwise the difference between them will be significant. We introduced the threshold $\lambda_{l}$ to test whether the difference is significant or not. When $\overline{f_{T}^{l}}$ falls in the region $\left[\overline{f_{T}^{l}}-\lambda_{l}, \overline{f_{T}^{l}}+\lambda_{l}\right]$, the difference is not significant. Because the $f_{k}^{l}$ is approximately normal, its mean is also approximately normal, then the threshold $\lambda_{1}$ can be given by

$$
\int_{f^{l}-\lambda_{l}}^{\overline{f^{l}}+\lambda_{l}} N\left(z ; \overline{f^{l}}, \sigma_{f^{l}}^{2} / K\right) d z=1-\alpha
$$

where $\alpha$ is the confidence level of the test.

when $\left|\overline{f_{T}^{l}}-\overline{f^{l}}\right| \geq \lambda_{l}$, the difference is significant and decision of the target motion state changed is made, the $K$ is the estimated onset time.

\section{Some experimental results}

Some experiments were carried out in a lake to illustrate the capability of the detector proposed in the paper. A small boat was used and its track is specified in the experiment. A broadband underwater recording buoy was used to record the boat's radiated noise. The position of the buoy 
and the boat was recorded by onboard GPS receiver. Some analysis had done using the recorded data after the experiment. Compared with the true motion state according to the GPS, the estimated result can be checked.

Firstly, a series of straight runs with two constant speeds was performed. Using the recorded data, four acoustic features are extracted, including NLS $\left(f^{1}\right)$, SWS $\left(f^{2}\right)$, largest line spectrum in the low frequency band $\left(f^{3}\right)$, and power in the frequency band $2000 \mathrm{~Hz}-2250 \mathrm{~Hz}\left(f^{4}\right)$. Table 1 gives the AFDI of the four features.

Table 1. the AFDI of the four features

\begin{tabular}{|c|c|c|c|c|c|}
\hline \multirow{2}{*}{} & \multicolumn{2}{|c|}{$v_{1}$} & \multicolumn{2}{|c}{$v_{2}$} & \multirow{2}{*}{$\gamma\left(f, v_{1}, v_{2}\right)$} \\
\cline { 2 - 5 } & $\overline{f\left(v_{1}\right)}$ & $\sigma_{f}^{2}\left(v_{1}\right)$ & $\overline{f\left(v_{2}\right)}$ & $\sigma_{f}^{2}\left(v_{2}\right)$ & \\
\hline$f^{1}$ & 8.14 & 10.54 & 14.98 & 11.08 & 2.16 \\
\hline$f^{2}$ & 2.167 & 0.0028 & 1.398 & 0.052 & 10.79 \\
\hline$f^{3}$ & 235.82 & 2785.47 & 255.38 & 573.12 & 0.11 \\
\hline$f^{4}$ & 0.124 & 0.0169 & 0.361 & 0.00048 & 3.23 \\
\hline
\end{tabular}

Then several maneuver runs was performed. For a special run, Fig. 2 gives its spectrum-time-recording in the frequency band $0-5000 \mathrm{~Hz}$, and Fig.3 gives the speed in the function of time according to the GPS. From the Fig.3, we can see the boat have significant speed change at 65s, and its spectrum also changed at 75s from the Fig.2.

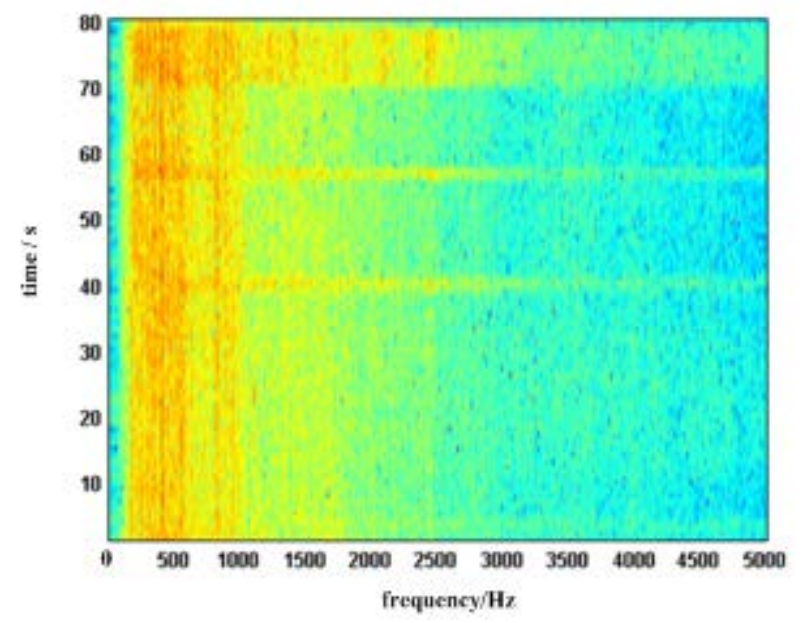

FIG. 2. The spectrum-time-recording in the frequency band $0-5000 \mathrm{~Hz}$

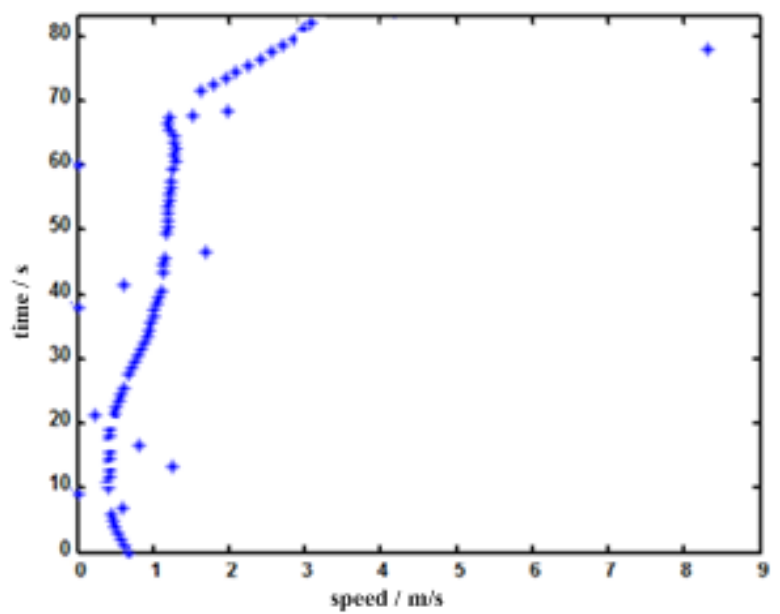

FIG. 3. the speed in the function of time according to the GPS 
According to the table 1, SWS $\left(f^{2}\right)$ has the largest AFDI, so we chose the SWS to detect the maneuver onset time. We use $T=1 \mathrm{~s}, K_{0}=30, m=0, S=10$, and $\alpha=0.8$ to develop the detector. Fig.4 gives the difference of feature value $\left|\overline{f_{T}^{2}}-\overline{f^{2}}\right|$ and threshold $\lambda_{2}$ in the function of time. From Fig. 4 we can see the target maneuver onset time is at $75 \mathrm{~s}$. The actual time according to the ship's GPS is at 70s. The proposed method is more rapid to estimate the maneuver onset time after the target maneuver occurs.

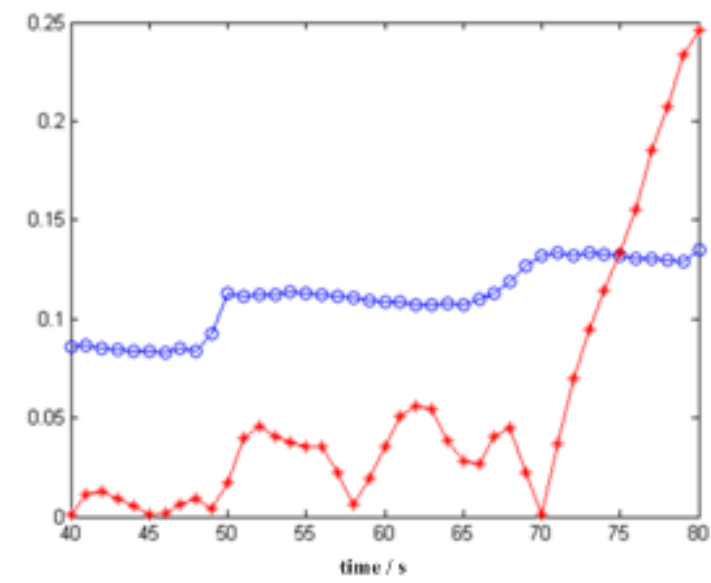

FIG. 4 the difference of feature value $\left|\overline{f_{T}^{2}}-\overline{f^{2}}\right|$ (denoted by asterisk) and threshold $\lambda_{2}$ (denoted by circle) in the function of time

\section{Conclusion}

Rapid and robust target maneuver onset time detection is crucial to obtain comparable target tracking accuracy. In this paper, we exploited the problem using the acoustic features of ship's radiated noise. By testing the significance of acoustic feature difference, an online method is proposed to estimate the target maneuver onset time. Experiment results show that the proposed method is more rapid to estimate the maneuver onset time after the target maneuver occurs.

\section{References}

[1] Nardone, S.C., Lindgren, A.G., and Gong, K.F.(1984) Fundamental Properties and Performance of Conventional Bearings-Only Target Motion Analysis. IEEE Transactions on Automatic Control, AC-29, 9(Sept. 1984), 775-787.

[2] M. J. Hinich. Tracking a Moving Vessel from Bearing Measurements. IEEE Journal of oceanic engineering, 1983, OE-8(3): 131-135

[3] Y. B. Shalom, X. R. Li,T Kirubarajan. Estimation with applications to tracking and navigation. Canada: John Wiley \& Sons, INC., 2001

[4] Tremois O. Bearing-Only Tracking for Maneuvering Source. IEEE Transaction on Aerospace and Electronic Systems, 1998, 34(1): 179 191

[5] Passerieux J M, Van Cappel D. Optimal Observer Maneuver for Bearings-Only Tracking. IEEE Transaction on Aerospace and Electronic Systems, 1998, 34(3): 892 901

[6] J. Ru, V. P. Jilkov, X. R. Li, A. Bashi, Detection of target maneuver onset, IEEE Transactions on Aerospace and Electronic Systems 45(2): 536-554

[7] E. J. Hughes, M. Leyland, Target maneuver detection using radar glint, Electronics Letters 34(17): 1695-1696

[8] C. Yang, E. Blasch, Estimating target range-Doppler image slope for maneuver indication, in: Proceedings of the SPIE Conference on Signal Processing, Sensor Fusion, and Target Recognition XVII, vol. 6968, March 2008, Orlando, FL, USA, pp.696808.1-696808.12. 